CME in preterm infants is limited; optimizing its characterization is a critical step in understanding its impact on vision. METHODS/ STUDY POPULATION: In this IRB-approved protocol, 118 preterm infants were imaged in the Duke intensive care nursery (ICN) with a novel lightweight, hand-held, high-speed, SS-OCT system following routine clinical eye exams. SS-OCT images were deidentified, automatically segmented using custom software (DOCTRAP), measured for several retinal layer thicknesses, and reviewed by masked expert graders for the presence and severity of CME. Reliability of SS-OCT measures will be assessed, and the association between CME status and retinal layer thicknesses will be calculated using logistic regression modeling. RESULTS/ ANTICIPATED RESULTS: The prevalence of CME overall and by severity will be calculated. The distribution of several retinal layer thicknesses will be reported and compared by infant CME status and, when edema is present, by CME severity. Reproducibility and repeatability will be reported for objective variables, and intra-grader and inter-grader agreement will be reported for subjective variables. Multivariate logistic regression coefficients and odds ratios will be calculated for each retinal layer thickness variable. DISCUSSION/ SIGNIFICANCE OF IMPACT: This study will use a novel SSOCT system to identify retinal thickness measures that may be objective markers of CME status. This will refine the characterization of CME and provide a framework for correlating CME with functional outcomes like visual acuity. CONFLICT OF INTEREST DESCRIPTION: SC and CT have unlicensed patents on relevant technologies. CT receives royalties from Alcon and Hemosonics and consultation fees from EMMES.

White Matter Integrity in Hemodialysis Patients

Wesley Richerson ${ }^{1}$, Dawn Wolfgram² ${ }^{2}$, and Brian Schmit ${ }^{3}$ ${ }^{1}$ Medical College of Wisconsin; ${ }^{2}$ Medical College of Wisconsin Department of Medicine; ${ }^{3}$ Marquette University Biomedical Engineering

OBJECTIVES/GOALS: The purpose of this study is to understand how hemodynamics during dialysis in End-Stage Renal Disease (ESRD) patients on hemodialysis (HD) affect white matter health and how those effects cause cognitive impairments. METHODS/ STUDY POPULATION: We collected demographic data, comorbidities, intradialytic measurements of blood pressure and cerebral oximetry, cognitive measures in several domains using NIH Toolbox Cognition Battery, diffusion-weighted and anatomical MRIs for 20 participants on HD. Specific tracts were identified using tractography and were used to calculate the average DTI measurements in each tract. Regression analysis was used to examine the relationship between mean DTI measurements of white matter integrity and cognitive performance scores. In addition, we compared diffusion MRI and T1 anatomical images of 16 healthy age-matched controls from a previous study. RESULTS/ANTICIPATED RESULTS: In our cohort 18 participants had imaging data that could be used in the analysis. We found widespread decreases in DTI white matter integrity compared to healthy age-matched controls, mean wholebrain fractional anisotropy was .3218 in the HD cohort and .3472 in controls $p=.0018$. Decreased integrity was found in most of the tracts identified but more decreased in tracts implicated in cognition. Partial regression analysis identified significant relationships between the white matter integrity of the left superior longitudinal fasciculus and overall fluid cognitive performance, $\mathrm{R}=.5525$, $\mathrm{p}=.0174$ before multiple comparisons correction when controlling for differences due to age. DISCUSSION/SIGNIFICANCE OF IMPACT: We found a widespread decrease in white matter integrity and significant correlations between cognitive performance and specific tract integrity in our HD cohort using regions identified by tractography imaging analysis. This analysis shows that HD patients have decreased white matter health and identifies several tracts that are important for cognitive performance in HD patients.

\section{Precision Medicine}

A latent class analysis of seriously ill adults with multiple chronic conditions receiving palliative care

Komal Murali ${ }^{1}$, Gary Yu ${ }^{2}$, John D. Merriman ${ }^{2}$, and Abraham A. Brody ${ }^{2}$ ${ }^{1}$ New York University; ${ }^{2}$ New York University Rory Meyers College of Nursing

OBJECTIVES/GOALS: The purpose of this secondary data analysis was to identify latent subgroups of seriously ill adults based on multiple chronic conditions and mortality risk using the CCI. This study was conducted by performing a secondary analysis of data from a randomized controlled trial of seriously ill patients receiving palliative care. METHODS/STUDY POPULATION: A cross-sectional analysis of baseline CCI data was conducted. 381 seriously ill adults receiving palliative care were in the original study. Latent subgroups were identified based on the CCI by conducting a latent class analysis in MPlus. The LCA was modeled on each of the 19 disease items as binary latent predictor variables, an additional binary variable representing presence of any disease not accounted for by the CCI, and a final categorical variable representing the total CCI score divided based on clinically significant cutoffs including zero, low $(>=1-<2)$, moderate $(>=2-<5)$, and high CCI $(>=5)$. RESULTS/ANTICIPATED RESULTS: Three distinct latent subgroups were identified based on the CCI. Latent subgroup 1 included those with a low-moderate CCI consisting of MCC and nonMetastatic Cancers $(n=178)$, with $45 \%$ of this group having chronic obstructive pulmonary disease. The second two subgroups included individuals with a high CCI or a score greater than or equal to 5 . Latent subgroup $2(n=64)$ was comprised of individuals with MCC and non-metastatic cancer. Latent subgroup $3(n=139)$ included individuals with metastatic cancer. DISCUSSION/ SIGNIFICANCE OF IMPACT: In a sample of seriously ill adults with MCC, latent subgroups were identified consisting of individuals with low, moderate, or high CCI. The low to moderate CCI group consists of individuals with chronic conditions including COPD, congestive heart failure, myocardial infarction, cardiovascular disease. There were two subgroups with high CCI scores and the differentiating factor between the two subgroups was the presence of metastatic cancer in latent subgroup 3. The identification of latent subgroups sets the groundwork for further analyses to compare differences in symptom burden, quality of life, and functional status between groups. The findings have the potential to inform future studies seeking to better characterize seriously adults with MCC based on their disease burden and mortality risk. 